\title{
Performance of ILC applied to a flexible mechanical system
}

Johanna Wallén, Mikael Norrlöf, Svante Gunnarsson

Division of Automatic Control

E-mail: johanna@isy.liu.se, mino@isy.liu.se, svante@isy.liu.se

3rd November 2008

Report no.: LiTH-ISY-R-2869

Submitted to European Control Conference (ECC) 2009, Budapest, Hungary

Address:

Department of Electrical Engineering

Linköpings universitet

SE-581 83 Linköping, Sweden

WWW: http://WwW. control.isy.liu.se

AUTOMATIC CONTROL

REGLERTEKNIK

LINKÖPINGS UNIVERSITET

Technical reports from the Automatic Control group in Linköping are available from http://www. control.isy.liu.se/publications. 


\begin{abstract}
ILC is traditionally applied to systems where the controlled variable is the measured variable. However, in standard industrial robots only the motor angles are measured, while the control objective is to follow a tool path. A modern industrial robot is flexible, and assuming that the mechanical flexibilities are concentrated to the robot joints (elastic gearboxes), a flexible two-mass model can be used to describe a single joint. A P-ILC algorithm is applied to the two-mass model, based on only measured angle of the first mass (motor angle) or estimated angle for the second mass (tool angle). Robustness of the algorithm, and performance when model errors are introduced in the model, are discussed considering the error of the tool angle. First, it can be concluded for a flexible system that the characteristics of the motor-angle reference is essential for the resulting tool angle when the tool angle cannot be measured. Second, using an estimate of the tool angle instead of the explicit motor angle in the ILC update reduces the tool-angle error significantly.
\end{abstract}

Keywords: Iterative learning control; Robotics; Robust control 


\title{
Performance of ILC applied to a flexible mechanical system
}

\author{
Johanna Wallén, Mikael Norrlöf and Svante Gunnarsson
}

\begin{abstract}
ILC is traditionally applied to systems where the controlled variable is the measured variable. However, in standard industrial robots only the motor angles are measured, while the control objective is to follow a tool path. A modern industrial robot is flexible, and assuming that the mechanical flexibilities are concentrated to the robot joints (elastic gearboxes), a flexible two-mass model can be used to describe a single joint. A P-ILC algorithm is applied to the two-mass model, based on only measured angle of the first mass (motor angle) or estimated angle for the second mass (tool angle). Robustness of the algorithm, and performance when model errors are introduced in the model, are discussed considering the error of the tool angle. First, it can be concluded for a flexible system that the characteristics of the motor-angle reference is essential for the resulting tool angle when the tool angle cannot be measured. Second, using an estimate of the tool angle instead of the explicit motor angle in the ILC update reduces the tool-angle error significantly.
\end{abstract}

\section{INTRODUCTION}

Traditionally Iterative Learning Control (ILC) has been applied to systems where the controlled variable also is the measured variable. In industrial robot applications, as for example plasma cutting shown in Fig. 1, this is typically not the case. In a standard industrial robot only the motor angles are measured. However, the control objective is to follow a desired tool path. Obviously it is a problem that the controlled variables cannot be measured, which is confirmed from experiments performed with a robot, see for example [16], [17]. A modern industrial robot is flexible, due to

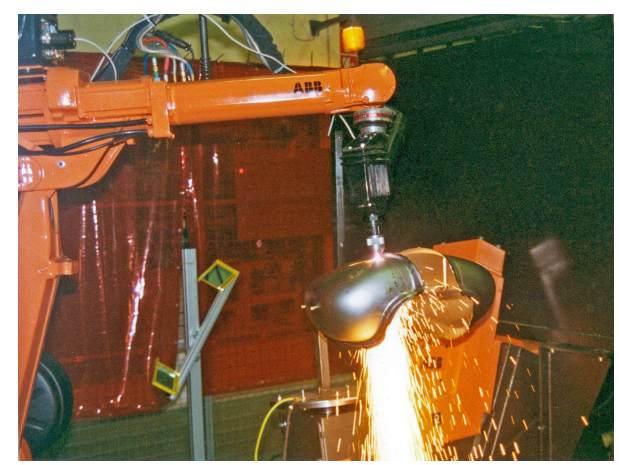

Fig. 1. A commercial industrial ABB robot IRB1400 performing plasma cutting [1]. Tool position and orientation cannot be measured due to practical and economical reasons. The control objective is however to follow a desired tool path, whereas the measured variables are the motor angles.

This work was supported by the Swedish Research Council (VR).

J. Wallén, S. Gunnarsson, and M. Norrlöf are with Division of Automatic Control, Department of Electrical Engineering, Linköping University, SE-58183 Linköping, Sweden, \{johanna, mino, svante\}@isy.liu.se a lower weight [4]. Assume that the mechanical flexibilities are concentrated to the robot joints (elastic gearboxes), the moment of inertia of the tool is constant, and that nonlinear phenomena like friction, backlash, etc., are neglected in the joints. Motivated from for example the first robot joint when the other joints are still, an approximate description of the joint is given by the flexible linear two-mass model shown in Fig. 2. In this paper the performance and robustness aspects of the problem when the controlled variable cannot be measured are studied using this idealised model of a single joint, where the motor angle (first mass) is the measured variable and the tool angle (second mass) is the controlled variable. The ILC algorithm is applied to the model, and the study considers three different cases:

1) The motor angle is the measured variable.

2) The tool angle is estimated by a Kalman filter from measurements of the motor angle.

3) The tool angle is the measured variable.

The ILC method was introduced in [2], [5], [6], and a summary of recent publications can be found in [3]. Robotics has always been an important field of application for ILC (see [11], [15] among others), because in many robotic applications the robot performs the same operation repeatedly starting from approximately the same initial conditions. One example is the plasma cutting application shown in Fig. 1.

In [10], [12], ILC is applied to flexible systems, but it is assumed that the tool variable is measurable. Here the twomass system is studied for the three cases mentioned above, for the two different input points of the ILC algorithm to the system where the ILC update is added to the motorangle reference or the tool-angle reference. The model errors; parameter variations in tool inertia $J_{a}$ and spring constant $k$, are introduced into the model, motivated by real model uncertainties found in industrial robot applications. Finally it is discussed how the robustness of the ILC algorithm can be related to the performance of the overall system when the tool angle in the two-mass system is considered.

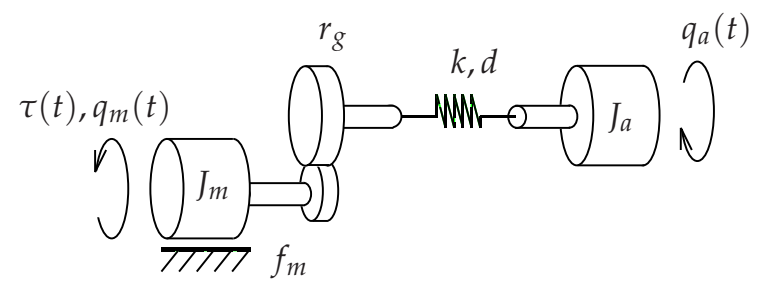

Fig. 2. A flexible two-mass model of a single robot joint, characterised by spring $k$, damper $d$, viscous friction $f_{m}$, gear ratio $r_{g}$, moments of inertia $J_{m}, J_{a}$, torque $\tau(t)$, motor angle $q_{m}(t)$ and tool angle $q_{a}(t)$. 


\section{PROBLEM DESCRIPTION}

\section{A. Two-mass joint model and controller}

The idealised model of a single robot joint is given by the flexible two-mass model, see Fig. 2,

$$
\begin{aligned}
J_{m} \ddot{q}_{m}(t)= & -f_{m} \dot{q}_{m}(t)-r_{g} k\left(r_{g} q_{m}(t)-q_{a}(t)\right) \\
& -r_{g} d\left(r_{g} \dot{q}_{m}(t)-\dot{q}_{a}(t)\right)+k_{\tau} \tau(t), \\
J_{a} \ddot{q}_{a}(t)= & k\left(r_{g} q_{m}(t)-q_{a}(t)\right)+d\left(r_{g} \dot{q}_{m}(t)-\dot{q}_{a}(t)\right),
\end{aligned}
$$

characterised by spring $k$, stiffness damping $d$, viscous friction $f_{m}$, gear ratio $r_{g}$, moments of inertia $J_{m}, J_{a}$, torque $\tau(t)$, motor angle $q_{m}(t)$ and tool angle $q_{a}(t)$. The parameter values used in the simulation are presented in Table I, and are from the study [8] of a laboratory-scale robot arm with some minor changes. Introducing the states

$$
x(t)=\left(\begin{array}{llll}
q_{a}(t) & \dot{q}_{a}(t) & q_{m}(t) & \dot{q}_{m}(t)
\end{array}\right)^{T},
$$

gives a state-space description of the system (1). The transfer functions

$$
\begin{aligned}
Q_{m}(s) & =G_{m}(s) \tau(s), \\
Q_{a}(s) & =G_{a}(s) Q_{m}(s),
\end{aligned}
$$

can then be derived. In Fig. 3 a block diagram of the controlled system with the ILC update is illustrated for case A, where $u_{k, A}(t)$ is added to $r_{m}(t)$ and case $\mathrm{B}$, where $u_{k, B}(t)$ is added to $r_{a}(t)$. Fairly moderate requirements for the feedback can be chosen, since the desired servo performance is planned to be achieved using the ILC algorithm. Here a PD controller including a low-pass filter is used, giving the transfer function

$$
F(s)=K_{p}+\frac{K_{d} s}{1+T_{f} s},
$$

where

$$
K_{p}=5, \quad K_{d}=0.2, \quad T_{f}=0.1 .
$$

The controller is implemented in discrete time in the simulation. Further details can be found in [16].

TABLE I

PARAMETERS USED IN THE FLEXIBLE TWO-MASS MODEL IN FIG. 2.

\begin{tabular}{|c|c|c|c|c|c|c|c|}
\hline$T_{s}$ & $r_{g}$ & $J_{m}$ & $J_{a}$ & $k$ & $d$ & $f_{m}$ & $k_{\tau}$ \\
\hline \hline 0.01 & 0.2 & 0.0021 & 0.0991 & 8 & 0.0924 & 0.0713 & 0.122 \\
\hline
\end{tabular}

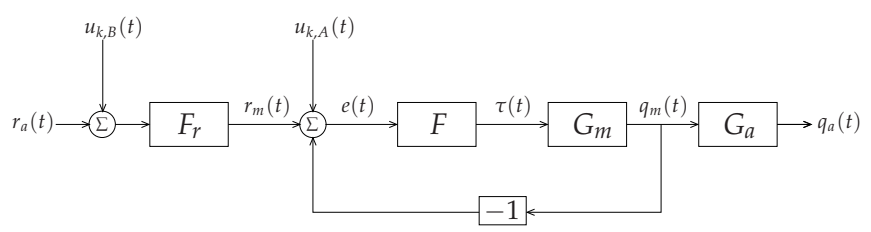

Fig. 3. The controlled system illustrated by $G_{m}$ and $G_{a}$; transfer function from motor torque to motor angle $q_{m}(t)$ and motor angle to tool angle $q_{a}(t)$, respectively. $F$ represents the feedback controller. The reference $r_{a}(t)$ is filtered by $F_{r}$ to give the motor-angle reference $r_{m}(t)$. The ILC input $u_{k}(t)$ at iteration $k$ is for case A added to $r_{m}(t)$ and for case B added to $r_{a}(t)$.
The system is driven by a tool-angle reference $r_{a}(t)$, which generally is given by the application. Here it is chosen as a filtered step. From Fig. 3 it can be seen that the motor-angle reference $r_{m}(t)$ is

$$
r_{m}(t)=F_{r}(q) r_{a}(t)
$$

The motor angle $q_{m}(t)$ is controlled towards the motor-angle reference $r_{m}(t)$. To be able to achieve the corresponding tool angle $q_{a}(t)$, which is the controlled variable, the tool-angle reference $r_{a}(t)$ is given from the filter $F_{r}$,

$$
F_{r}(q)=\frac{1}{q} \frac{1}{G_{a, d}(q)} .
$$

The transfer function $G_{a, d}(q)$ is a sampled version of $G_{a}(s)$ for the nominal case with no model errors introduced in the model. Finally, the factor $1 / q$ is introduced to make $F_{r}(q)$ proper. Fig. 4 shows the tool-angle reference $r_{a}(t)$ used in the paper and the motor-angle reference $r_{m}(t)$ from (6).

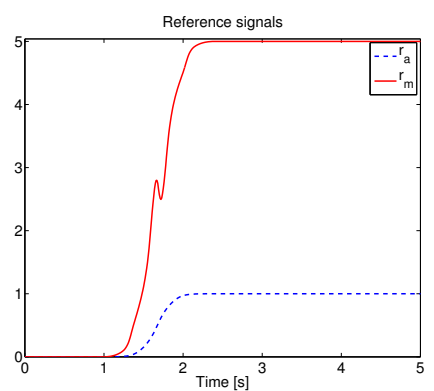

Fig. 4. The tool-angle reference $r_{a}(t)$ used in the paper and the corresponding motor-angle reference $r_{m}(t)$ given from (6).

\section{B. Estimation of tool angle}

Using motor torque $\tau(t)$ and measurements of the motor angle $q_{m}(t)$, the tool angle $q_{a}(t)$ can be estimated by a discrete-time Kalman filter. In the estimation, the following state-space description is used

$$
\begin{aligned}
x(t+1) & =A x(t)+B \tau(t)+w(t), \\
y(t) & =C x(t)+v(t),
\end{aligned}
$$

which is the discrete-time state-space description of the system (1) with the states (2). Process noise $w(t)$ and measurement noise $v(t)$ are added to the discrete-time state-space description. It is assumed that $w(t)$ and $v(t)$ are uncorrelated white noise with

$$
E\left(w(t) w^{T}(t)\right)=Q, \quad E\left(v(t) v^{T}(t)\right)=R .
$$

The covariance matrices $Q$ and $R$ are tuning parameters of the Kalman filter and the relation is a compromise between a fast filter and a filter sensitive to noise, see for example [9] for a thorough discussion of the Kalman filter. The parameter values of the covariance matrices have been tuned by minimising $\sum_{i}\left\|q_{a}(t)-\hat{q}_{a, i}(t)\right\|_{2}$ for the cases $i=\left\{\right.$ nominal, $\left.0.5 k, 2 k, 0.5 J_{a}, 2 J_{a}\right\}$, which are the cases investigated further in this paper. The tuning results in

$$
Q=\operatorname{diag}\left(\begin{array}{llll}
0 & 1 & 0 & 10^{-8}
\end{array}\right), \quad R=\left(10^{-8}\right) .
$$




\section{ILC ALGORITHM}

It is assumed that the general description for a linear discrete-time system can be formulated as

$$
y_{k}(t)=T_{r}(q) r(t)+T_{u}(q) u_{k}(t),
$$

where the ILC input signal and the output from the system are $u_{k}(t)$ and $y_{k}(t)$, respectively, $k$ denotes iteration number and $r(t)$ is the reference input. The signals are defined on a finite time interval $t=0, \ldots, N$ with $N$ number of samples. Finally, $T_{r}(q)$ and $T_{u}(q)$ are stable discrete-time filters. System and measurement disturbances are not included here, but can be easily treated in this framework, see for example [13].

The update for a general first-order ILC algorithm is

$$
u_{k+1}(t)=Q(q)\left(u_{k}(t)+L(q) e_{k}(t)\right)
$$

where the linear filters $Q(q)$ and $L(q)$ are possibly noncausal. The error

$$
e_{k}(t)=r(t)-y_{k}(t)
$$

is the difference between reference and measured output at iteration $k$. The relations (11) - (12) are in the sequel called an ILC system.

The update equation (12) implies the standard frequencydomain convergence criterion, see [14],

$$
\left|1-L\left(e^{i \omega}\right) T_{u}\left(e^{i \omega}\right)\right|<\left|Q^{-1}\left(e^{i \omega}\right)\right|, \quad \forall \omega,
$$

where $T_{u}$ is given from (11). From (14) it is clear that the stability region of the algorithm can be enlarged by choosing $\left|Q\left(e^{i \omega}\right)\right|$ small. The result is that the final error does not converge to zero, as is shown in, for instance, [7].

\section{SIMULATION RESULTS}

In the simulation the measurements used in the ILC update (12) - (13) versus input points of the ILC algorithm into the system are studied. To evaluate the performance and robustness of the ILC system, model errors are introduced in the two-mass model (1).

\section{A. Categorisation of the cases}

The conditions can be divided into:

1) $q_{m}(t)$ is the measured variable, which gives that the ILC update uses $r_{m}(t)-q_{m}(t)$, where $r_{m}(t)$ is given from $r_{a}(t)$ by the relation (6).

2) $q_{a}(t)$ is estimated by a Kalman filter from measurements of $q_{m}(t)$, resulting in $\hat{q}_{a}(t)$. The ILC update uses $r_{a}(t)-\hat{q}_{a}(t)$.

3) $q_{a}(t)$ is the measured variable. The ILC update uses $r_{a}(t)-q_{a}(t)$. This ideal case is compared to the cases above.

The input points of the ILC algorithm into the system are:

A) The ILC update $u_{k, A}(t)$ is added to $r_{m}(t)$, see Fig. 3 .

B) The ILC update $u_{k, B}(t)$ is added to $r_{a}(t)$, see Fig. 3.

Combining measurement cases $1,2,3$ and input points A,B give various setups for the ILC system. For example, case $2 \mathrm{~A}$ means that the estimated tool angle $\hat{q}_{a}(t)$ is used to compute the error (13), which is used in the ILC update (12). The update $u_{k, A}(t)$ is then added to $r_{m}(t)$, as in Fig. 3. The tool angle $q_{a}(t)$ is used as an evaluation variable that should follow the desired tool trajectory for all cases.

For the cases studied a P-type ILC algorithm is designed, where $L=\gamma q^{\delta}$. The $Q$ filter is chosen as a low-pass secondorder Butterworth filter with a cutoff frequency $\omega_{n}$ above the bandwidth of the controlled system. The reason for this is that with a cutoff frequency below the bandwidth only the low-frequency components of the error are corrected for, while the characteristics associated with the resonance frequency is not taken into account. The $Q$ filter is applied by filtering the signal forwards and backwards to give zerophase characteristics. The ILC design parameters $\omega_{n}, \delta, \gamma$ for each case result from tuning of the ILC algorithm ad hoc, such that the tool-angle error $\left\|e_{a}(t)\right\|_{2}$ is reduced to around $4 \%$ after the ILC algortihm has converged and that the error reduction is achieved within approximately 50 iterations, which is illustrated in Fig. 5. The frequency-domain convergence criterion (14) is also satisfied for the resulting ILC design parameters. Broadly the cutoff frequency $\omega_{n}$ of the $Q$ filter decides the level of the final error, while $\gamma$ is associated with convergence speed, which means that the tuning is a tradeoff between robustness and performance.

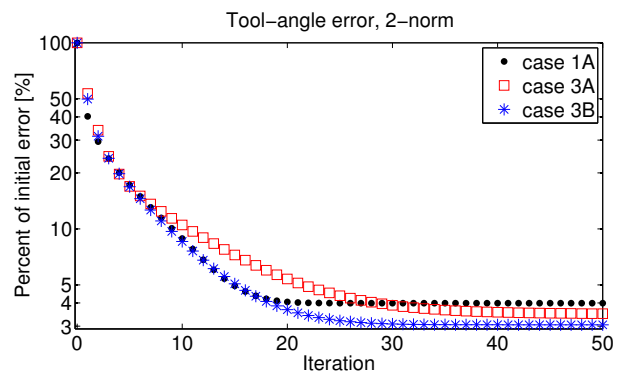

Fig. 5. Tuning of the algorithm for the nominal cases $1 \mathrm{~A}, 3 \mathrm{~A}, 3 \mathrm{~B}$. The tool-angle error is reduced to around $4 \%$ of the initial error (no ILC) within approximately 50 iterations.

\section{B. ILC applied to motor-angle reference, using motor-angle error (case 1A)}

Case $1 A$ means that the ILC algorithm uses the motorangle error $e_{m}(t)=r_{m}(t)-q_{m}(t)$ and the ILC update $u_{k, A}(t)$ is applied to the system according to the structure shown in Fig. 3. The motor-angle reference is described by (6) and is a filtered tool-angle reference. The ILC design parameters are $\omega_{n}=10 \mathrm{~Hz}, \gamma=0.9$ and $\delta=10$.

In Fig. 7 the motor angle is shown when the ILC algorithm has converged. The resulting behaviour when a model error, a spring constant equal to $1.5 k$, is introduced into the model is also shown. From the left figure it can be seen that the ILC algorithm is robust, since the motor-angle errors $e_{m}(t)$ are significantly reduced both for the nominal case and when model errors are introduced, compared to the initial motorangle error in Fig. 6 when no ILC algorithm is applied. To the right the resulting motor angle $q_{m}(t)$ is compared to the initial value. It can be seen that the motor angle follows $r_{m}(t)$ very well, also in the case of model errors. 
Fig. 8 shows the tool angle. The ILC algorithm reduces the tool-angle error significantly for the nominal system. The tool angle is however worsen compared to the nominal case in Fig. 6 when model errors are introduced, as expected. The performance is sensitive with respect to the pre-filter $F_{r}$ the tool angle converges, but towards the incorrect signal.

To summarise, the ILC algorithm using motor-angle errors and updating the motor-angle reference is robust with respect to model errors. However, the tool performance is sensitive with respect to the pre-filter $F_{r}$, and the tool angle converges towards the incorrect signal when model errors are introduced. The motor-angle reference is thereby essential for the resulting tool angle after convergence.
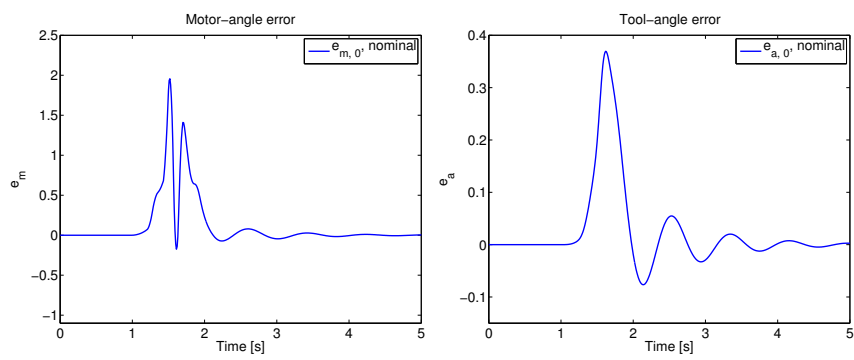

Fig. 6. Initial motor-angle error $e_{m}(t)$ and tool-angle error $e_{a}(t)$ for the nominal case when no ILC algorithm is applied to the system.
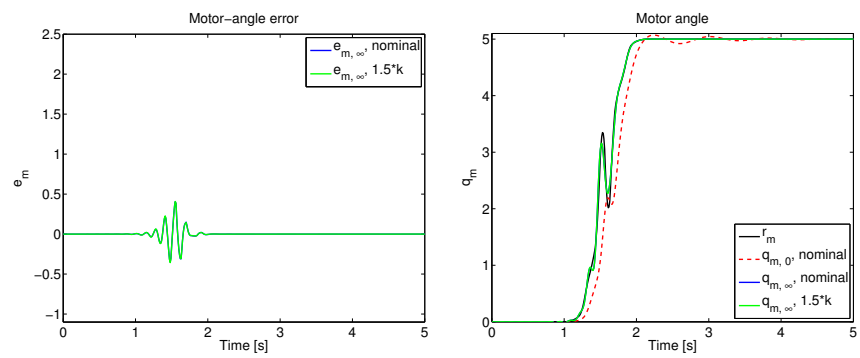

Fig. 7. Case 1A: Resulting motor angle when the ILC algorithm has converged (denoted $\infty$ ). The ILC algorithm reduces the motor-angle errors both in the nominal case and the case with model errors $(1.5 k)$, compared to the behaviour with no ILC applied.
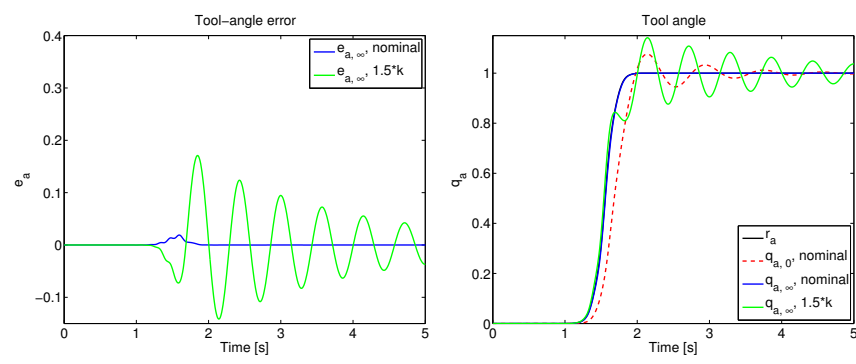

Fig. 8. Case 1A: Resulting tool angle when the ILC algorithm has converged (denoted $\infty$ ). The ILC algorithm reduces the tool-angle error, but for the case with model errors $(1.5 k)$, the behaviour of the tool is worsen compared to the nominal case.
C. ILC applied to motor-angle reference, using tool-angle error (case $2 A, 3 A$ )

The ILC design parameters are $\delta=20, \gamma=2.0$ (possible, since $\left.\left|T_{u}(0)\right|=0.2\right)$, and $\omega_{n}=8 \mathrm{~Hz}$. The ILC update $u_{k, A}(t)$ is added to the motor-angle reference $r_{m}(t)$ as in Fig. 3.

Case 2A: To improve the result in Sec. IV-B, the tool angle $\hat{q}_{a}(t)$ is estimated from measurements of the motor angle by the Kalman filter in Sec. II-B. The estimate is then used by the ILC algorithm. See also [8], where measurements of motor angle and acceleration of the tool form an estimate of the tool angle, which is used in the ILC update.

In Fig. 9 the motor-angle error $e_{m}(t)$ is shown to the left. The resulting motor angle $q_{m}(t)$ to the right is clearly improved for the nominal case, compared to the initial error in Fig. 6. This is expected, because the ILC update using the tool-angle error also reduces the motor-angle errors when no model errors are introduced in the model. When the model error $1.5 k$ is introduced, it can be seen that the motor-angle error is reduced, but $q_{m}(t)$ does not follow $r_{m}(t)$ exactly.

The main result of the paper is shown in Fig. 10, where the tool-angle errors and the resulting tool angle are illustrated. The tool-angle error is significantly reduced both for the nominal case and in the case of model errors, compared to the initial error without ILC shown in Fig. 6. See also the large improvement of the tool angle compared to the case $1 \mathrm{~A}$ in Fig. 8, where only the motor-angle error is used in the ILC update. The error reduction is notable, since the ILC update uses the estimated tool angle $\hat{q}_{a}(t)$ instead of the measured tool angle $q_{a}(t)$, with the estimation error $q_{a}(t)-\hat{q}_{a}(t)$ shown in Fig. 10 .
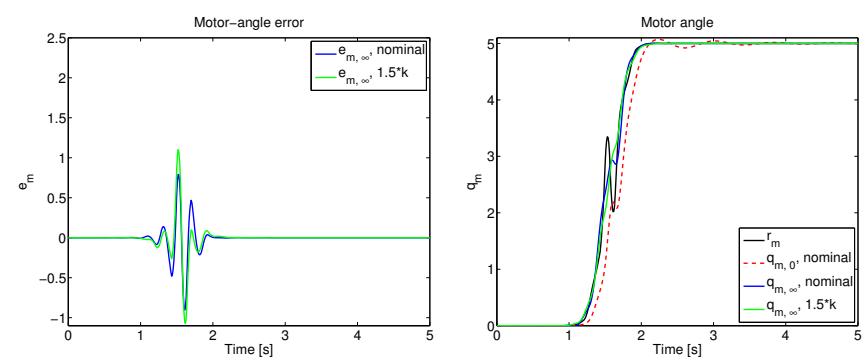

Fig. 9. Case 2A: Resulting motor angle when the ILC algorithm has converged (denoted $\infty$ ). The ILC algorithm reduces the motor-angle errors for the nominal case, but for the case with model error $(1.5 k)$ the reduction is smaller. It is however reduced compared to the size of the initial error.

Case 3A: The ILC update is now based on the measured tool angle $q_{a}(t)$. This case should be seen as an illustration to what can ideally be achieved, compared to the case $2 \mathrm{~A}$. Fig. 11 shows the tool-angle error and the resulting tool angle. Comparing case $2 \mathrm{~A}$ in Fig. 10 to the case $3 \mathrm{~A}$ in Fig. 11, one can see that it is possible to reduce the toolangle error practically as much using the estimate $\hat{q}_{a}(t)$ as using the measured tool angle $q_{a}(t)$. It can also be noted that both the ILC algorithm and the performance of the tool after convergence are robust with respect to model errors. 


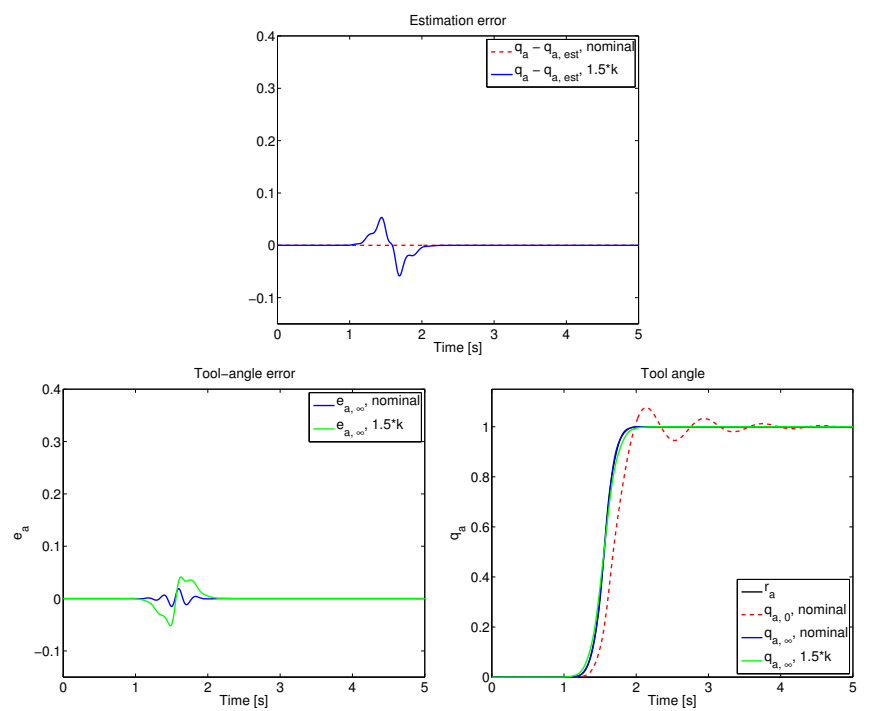

Fig. 10. Case 2A: Resulting tool angle when the ILC algorithm has converged (denoted $\infty$ ). The tool-angle error is significantly reduced both for the nominal case and and in the case of model errors $(1.5 k)$, despite the estimation error in the upper figure.
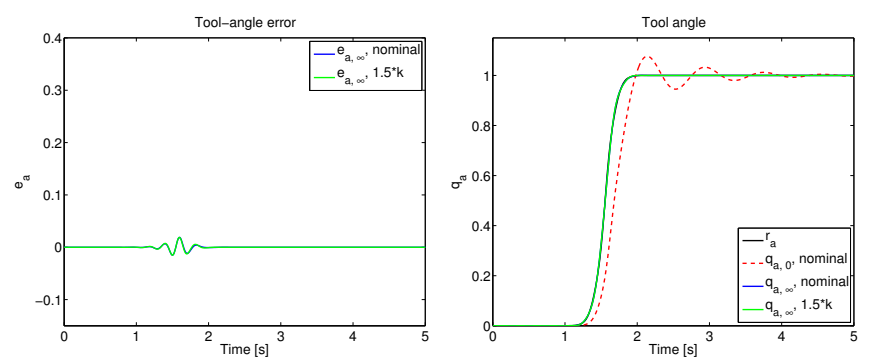

Fig. 11. Case 3A: Resulting tool angle when the ILC algorithm has converged (denoted $\infty$ ). The tool-angle error is significantly reduced, both for the nominal case and the case of the model error (1.5k). This case shows what ideally be achieved, compared to the case $2 \mathrm{~A}$.

\section{ILC applied to tool-angle reference, using tool-angle} error (case 2B, 3B)

The ILC design variables are chosen as $\delta=12, \gamma=0.6$, and $\omega_{n}=6 \mathrm{~Hz}$.

Case 2B: The ILC update $u_{k, B}(t)$ is based on the error $e_{a}(t)=r_{a}(t)-\hat{q}_{a}(t)$ and is added to the tool-angle reference $r_{a}(t)$, as is shown in Fig. 3. The resulting tool angle when the ILC algorithm has converged can be seen in Fig. 12. The results are similar to those in previous section - using the estimate $\hat{q}_{a}(t)$ in the ILC update significantly reduces the tool-angle error after the ILC algorithm has converged, compared to the initial tool-angle error shown in Fig. 6. This is the case even though large model errors are introduced.

The difference between case $2 \mathrm{~A}$ and $2 \mathrm{~B}$ is that the case $2 \mathrm{~B}$, where the ILC algorithm applied to the tool-angle reference using the tool-angle error, can handle smaller model errors compared to the case 2A. It can be explained by Fig. 3 where it can be seen that the the design relies on a feed-forward filter $F_{r}$, see (6), which is based on the inverse of the nominal system $G_{a}$. Therefore this structure of the ILC system is more sensitive to model errors.

Case 3B: The ILC update is based on the measured tool angle $q_{a}(t)$ and shows what can ideally be achieved, compared to the case $2 \mathrm{~B}$. The resulting tool angle is shown in Fig. 13. When comparing the results from Fig. 12 it can be seen that the resulting tool angle for case $2 \mathrm{~B}$ is close to what can ideally be achieved in case $3 \mathrm{~B}$, also in the case of model errors.

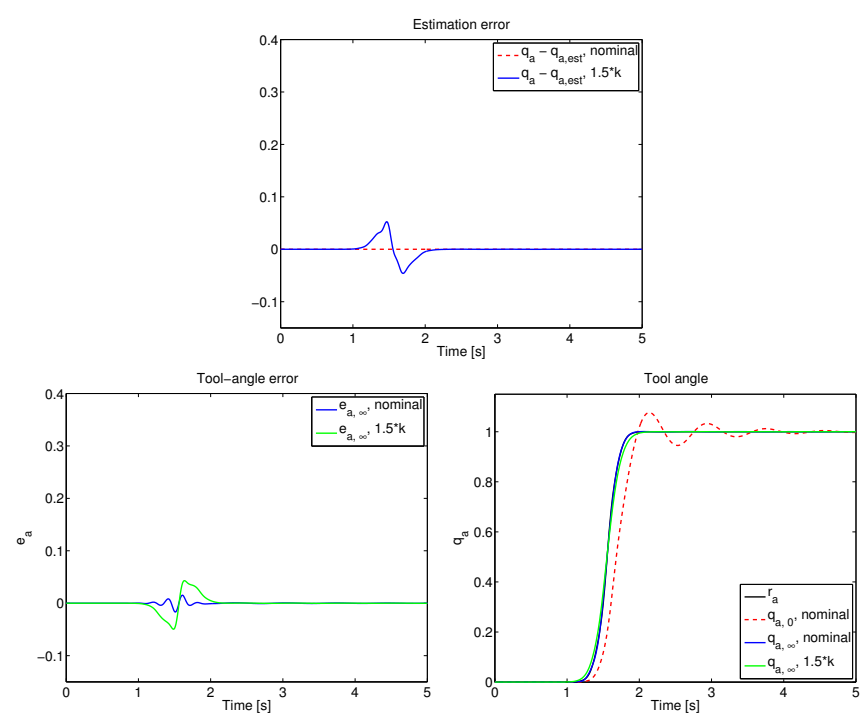

Fig. 12. Case 2B: Resulting tool angle when the ILC algorithm has converged (denoted $\infty$ ). The ILC algorithm reduces the tool-angle errors for the ideal case and also when the model error $(1.5 k)$ is introduced.
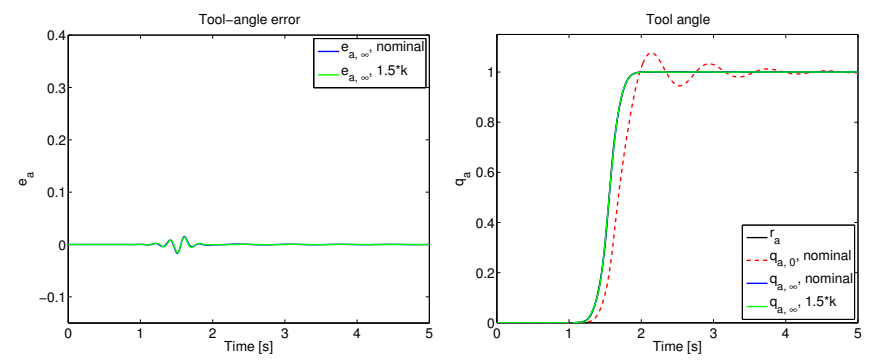

Fig. 13. Case 3B: Resulting tool angle when the ILC algorithm has converged (denoted $\infty$ ). The result of the nominal case is compared to when the model error $(1.5 k)$ is introduced. This case shows what ideally be achieved, compared to the case $2 \mathrm{~B}$.

\section{E. Performance of the tool}

The parameter variations in moment of inertia $J_{a}$ of the tool and spring constant $k$ correspond to the cases where the mass of the tool (including the load) is incorrect and the stiffness of the gearbox is uncertain, respectively.

One example of requirements for the tracking accuracy of the tool-angle is an overshoot of less than $1 \%$ when the ILC algorithm has converged. In Table II the maximum model errors for the parameters $J_{a}$ and $k$ that satisfy this 
requirement are shown. These values should be compared to the results shown in the figures in Secs. IV-B to IV-D, where it is illustrated how the model error $1.5 k$ affects the behaviour of the tool angle.

The robust performance of the complete system for the case 1A (the ILC algorithm update is based on the motorangle error) is clearly limited by the specification of the tool behaviour. In practice it is therefore not the robustness of the ILC algorithm that limits the actual model errors that can be introduced for case 1A. Instead it is the fact that the design relies on a feed-forward filter $F_{r}$ based on the inverse of the nominal system. For the cases $2 \mathrm{~A}$ and $3 \mathrm{~A}$, where the ILC update is based on the tool-angle error $e_{a}(t)=r_{a}(t)-\hat{q}_{a}(t)$, much larger model errors can be introduced and still having an overshoot of the tool angle of less than $1 \%$ when the ILC algorithm has converged. The model errors are instead limited by the frequency-domain convergence criterion (14).

From Table II it can also be seen that case $3 \mathrm{~A}$ can handle larger model errors in most cases without violating the convergence criterion (14), compared to case 2A, as was discussed in Sec. IV-D.

TABLE II

MAXIMUM MODEL ERRORS FOR THE PARAMETERS $J_{a}$ AND $k$, GIVING A TOOL-ANGLE BEHAVIOUR SATISFYING $1 \%$ OVERSHOOT.

\begin{tabular}{|l||c|c|}
\hline Conditions & Deviation in $J_{a}$ & Deviation in $k$ \\
\hline \hline ILC on motor side, & $+3.1 \%$ & $+2.8 \%$ \\
using $e_{m}$ (case 1A) & $-2.7 \%$ & $-2.9 \%$ \\
\hline ILC on motor side, & $+14 \%^{1}$ & $+372 \%^{1}$ \\
using estimated $e_{a}$ (case 2A) & $-80 \%^{1}$ & $-19 \%$ \\
\hline ILC on tool side, & $+19 \%^{1}$ & $+53 \%{ }^{1}$ \\
using estimated $e_{a}$ (case 2B) & $-34 \%^{1}$ & $-16 \%^{1}$ \\
\hline
\end{tabular}

${ }^{1}$ Maximum model error where the frequency-domain convergence criterion (14) is satisfied.

\section{CONCLUSIONS}

Iterative learning control (ILC) is applied to a flexible two-mass system representing an idealised model of a single robot joint, where the angle of the motor (first mass) is the measured variable and the angle of the tool (second mass) is the controlled variable. This problem is relevant, since in standard industrial robots only the motor angles are measured while the control objective is to follow a desired tool path. A P-type ILC design method has been studied in simulations, for two different input points of the ILC algorithm to the system; the ILC update is added to the motor-angle reference or the tool-angle reference.

First, it can be concluded that an ILC update based on an estimate of the tool angle reduces the final tool-angle error after convergence significantly compared to the initial error without ILC. This is the case even though large model errors are introduced.

When only the motor-angle error is used in the ILC update, the tool converges towards an incorrect signal when model errors are introduced. The second conclusion is therefore that the ILC update based on the motor-angle error is more sensitive to model errors than when using an estimate of the tool angle in the update. This can be explained by the fact that the motor-angle reference is computed using the inverse of the nominal system relating motor angle to tool angle.

Third, the input points of the ILC algorithm plays a role in terms of how robust the ILC algorithm is when model errors are introduced. The reason for this is that the design relies on a feed-forward filter, when the ILC update is added to the tool-angle reference. This filter is based on the inverse of the nominal system relating motor angle to tool angle.

\section{REFERENCES}

[1] ABB, 2008. URL: http: / /www . abb. se, accessed October, 2008.

[2] S. Arimoto, S. Kawamura, and F. Miyazaki. Bettering operation of robots by learning. Journal Robot. Syst., 1(2):123-140, 1984.

[3] D. A. Bristow, M. Tharayil, and A. G. Alleyne. A survey of iterative learning control. IEEE Control Syst. Mag., pages 96-114, 2006.

[4] T. Brogårdh. Present and future robot control development - An industrial perspective. Annual Reviews in Control, 31(1):69-79, 2007.

[5] G. Casalino and G. Bartolini. A learning procedure for the control of movements of robotic manipulators. In IASTED Sym. Robot. Autom., pages 108-111, San Francisco, USA, May 1984.

[6] J. J. Craig. Adaptive control of manipulators through repeated trials. In Proc. American Control Conf., pages 1566-1572, San Diego, CA, June 1984.

[7] H. Elci, R. W. Longman, M. Q. Phan, J.-N. Juang, and R. Ugoletti. Simple learning control made practical by zero-phase filtering: Applications to robotics. IEEE Trans. Circuits Syst. I, Fundam. Theory Appl., 49(6):753-767, June 2002.

[8] S. Gunnarsson, M. Norrlöf, E. Rahic, and M. Özbek. On the use of accelerometers in iterative learning control of a flexible robot arm. Int. Journal Control, 80(3):363-373, March 2007.

[9] T. Kailath, A. H. Sayed, and B. Hassibi. Linear estimation. PrenticeHall, Inc, Upper Saddle River, NJ, USA, 2000.

[10] K. Kinoshita, T. Sogo, and N. Adachi. Adjoint-type iterative learning control for a single-link flexible arm. In Proc. IFAC World Congress, Barcelona, Spain, July 2002.

[11] R. W. Longman. Iterative learning control and repetitive control for engineering practice. Int. Journal Control, 73(10):930-954, July 2000.

[12] P. Lucibello, S. Panzieri, and G. Ulivi. Repositioning control of a two-link flexible arm by learning. Automatica, 33(4):579-590, 1997.

[13] M. Norrlöf. Iterative Learning Control: Analysis, Design and Experiments. Dissertations no. 653, Dept. Electr. Eng., Linköpings universitet, Sweden, October 2000.

[14] M. Norrlöf and S. Gunnarsson. Time and frequency domain convergence properties in iterative learning control. Int. Journal Control, 75:1114-1126, 2002.

[15] A. Tayebi. Adaptive iterative learning control for robot manipulators. Automatica, 40(7):1195-1203, July 2004.

[16] J. Wallén. On Kinematic Modelling and Iterative Learning Control of Industrial Robots. Licentiate thesis no. 1343, Dept. Electr. Eng., Linköpings universitet, Sweden, January 2008. Available: http://www.control.isy.liu.se/research/ reports/LicentiateThesis/Lic1343.pdf.

[17] J. Wallén, M. Norrlöf, and S. Gunnarsson. Arm-side evaluation of ILC applied to a six-degrees-of-freedom industrial robot. In Proc. IFAC World Congress, Seoul, Korea, July 2008. 


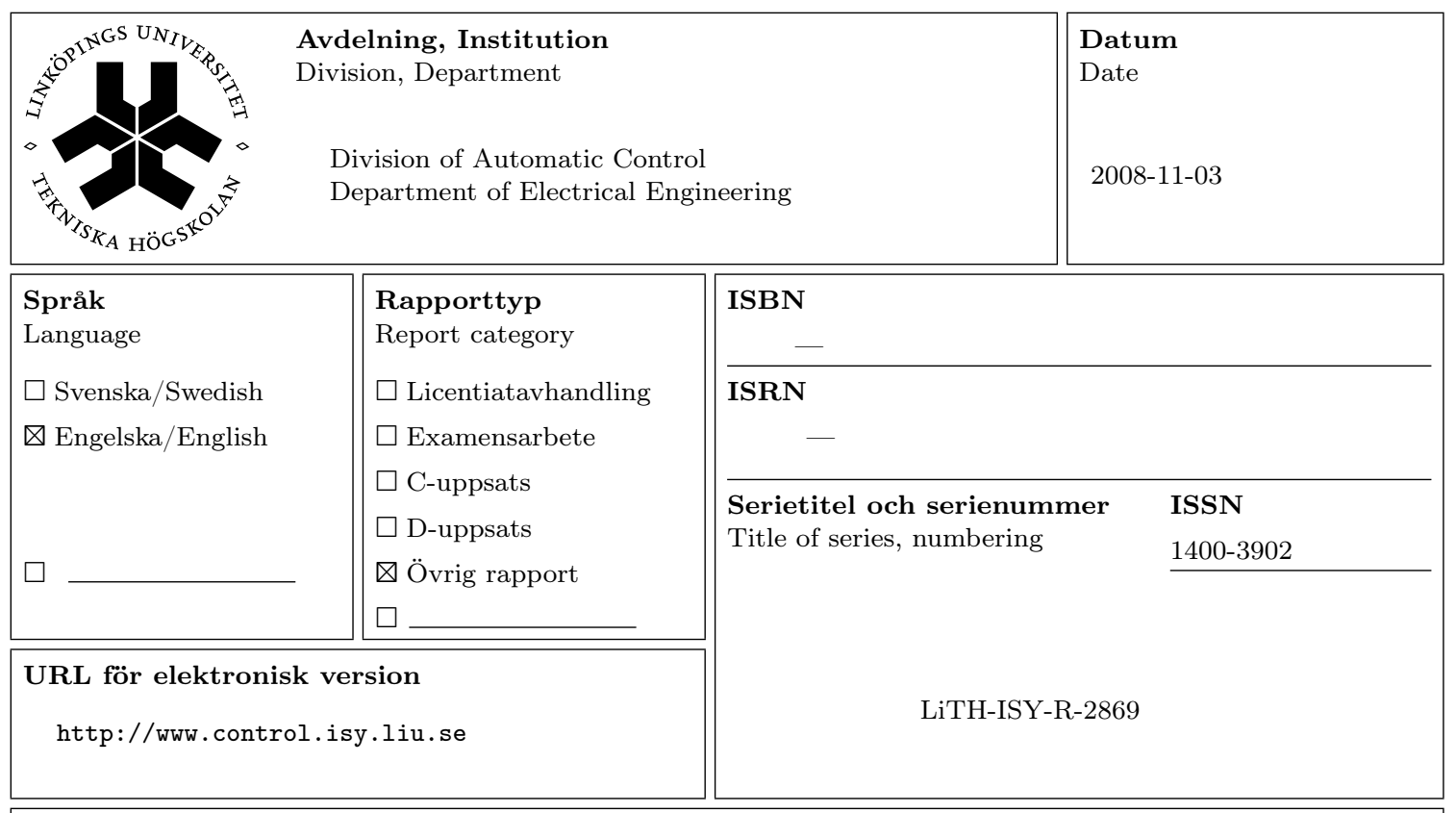

Titel Performance of ILC applied to a flexible mechanical system

Title

Författare Johanna Wallén, Mikael Norrlöf, Svante Gunnarsson

Author

\section{Sammanfattning}

Abstract

ILC is traditionally applied to systems where the controlled variable is the measured variable. However, in standard industrial robots only the motor angles are measured, while the control objective is to follow a tool path. A modern industrial robot is flexible, and assuming that the mechanical flexibilities are concentrated to the robot joints (elastic gearboxes), a flexible two-mass model can be used to describe a single joint. A P-ILC algorithm is applied to the two-mass model, based on only measured angle of the first mass (motor angle) or estimated angle for the second mass (tool angle). Robustness of the algorithm, and performance when model errors are introduced in the model, are discussed considering the error of the tool angle. First, it can be concluded for a flexible system that the characteristics of the motor-angle reference is essential for the resulting tool angle when the tool angle cannot be measured. Second, using an estimate of the tool angle instead of the explicit motor angle in the ILC update reduces the tool-angle error significantly. 